\title{
How to Create Exponential Decline in Car Use in Australian Cities
}

By Peter Newman, Jeff Kenworthy and Gary Glazebrook.

Curtin University and University of Technology Sydney.

Car dependent cities like those in Australia have always been increasing in car use. Measured as vehicle kms of travel or VKT this has been projected to go on increasing by every transport and planning agency in Australia (see Fig 1). Yet we have to reduce greenhouse gases by at least $50 \%$ by 2050 and maybe even $90 \%$. And the peak oil theorists show that oil supply will mean that must happen anyway. Most responses to how we will manage to do that can only imagine improving vehicle efficiency and changing fuels, they cannot see vehicle use going into the kind of exponential decline that would create such major change. 


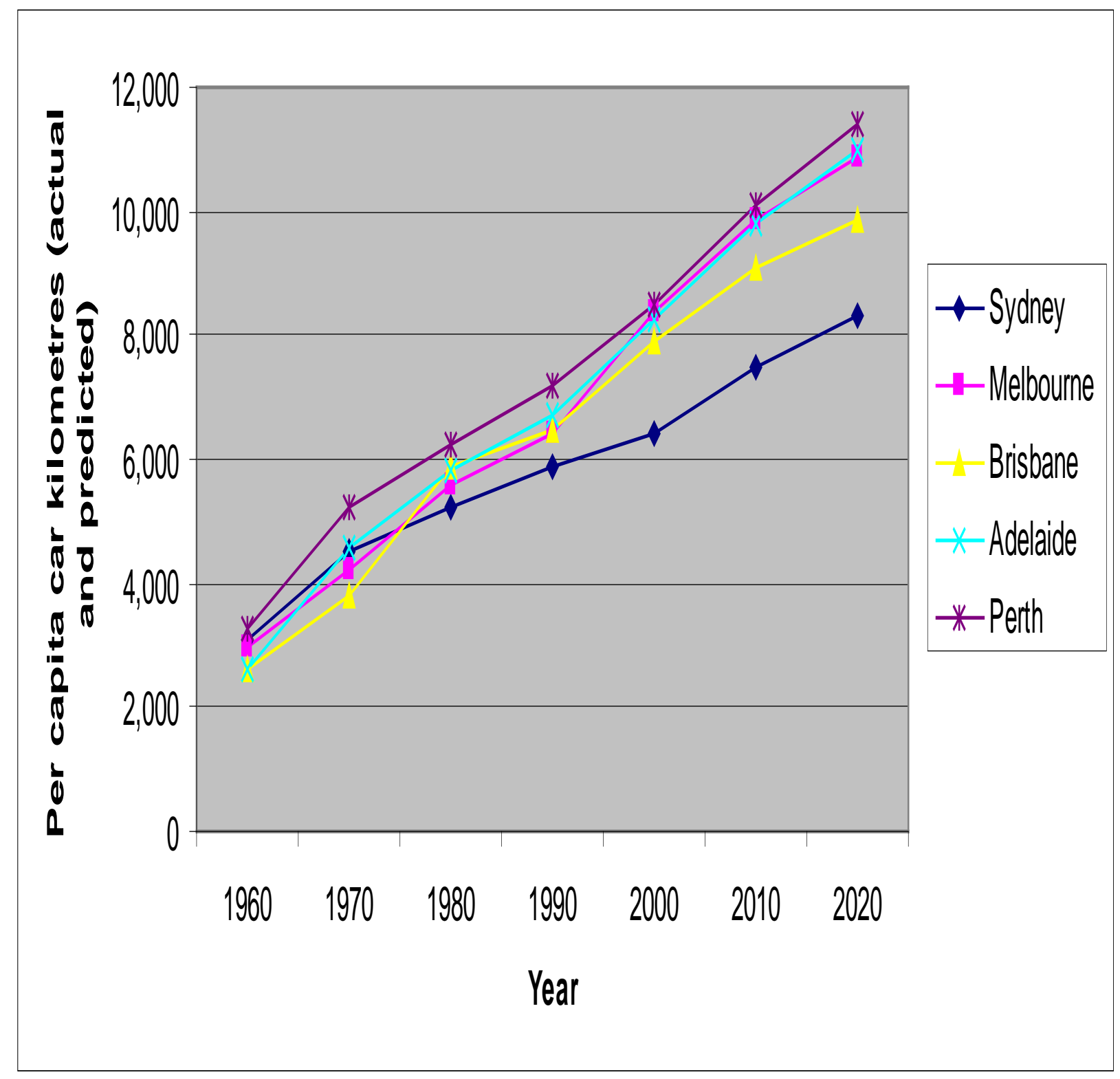

This paper will suggest how it is possible to imagine an exponential decline in car use in our cities that could lead to $50 \%$ less passenger kms driven in cars. The key mechanism is a quantitative leap in the quality of public transport whilst fuel prices continue to climb, accompanied by an associated change in land use patterns.

Figure 2 shows the relationship between car passenger kms and public transport passenger kms from the CUSP Global Cities Database. The most important thing about this relationship is that as the use of public transport increases linearly the car passenger kms decrease exponentially. This is due to a phenomenon called Transit Leverage whereby one pass $\mathrm{km}$ of transit use replaces between 3 and 7 pass $\mathrm{kms}$ in a car due to more direct travel (especially in trains), trip chaining (doing various other things like 
shopping or service visits associated with a commute), giving up one car in a household (a common occurrence that reduces many solo trips) and eventually changes in where people live as they prefer to live or work nearer transit.

\section{PUBLIC TRANSPORT PASSENGER KI LOMETRES PER CAPITA VERSUS CAR PASSENGER KI LOMETRES PER CAPITA}

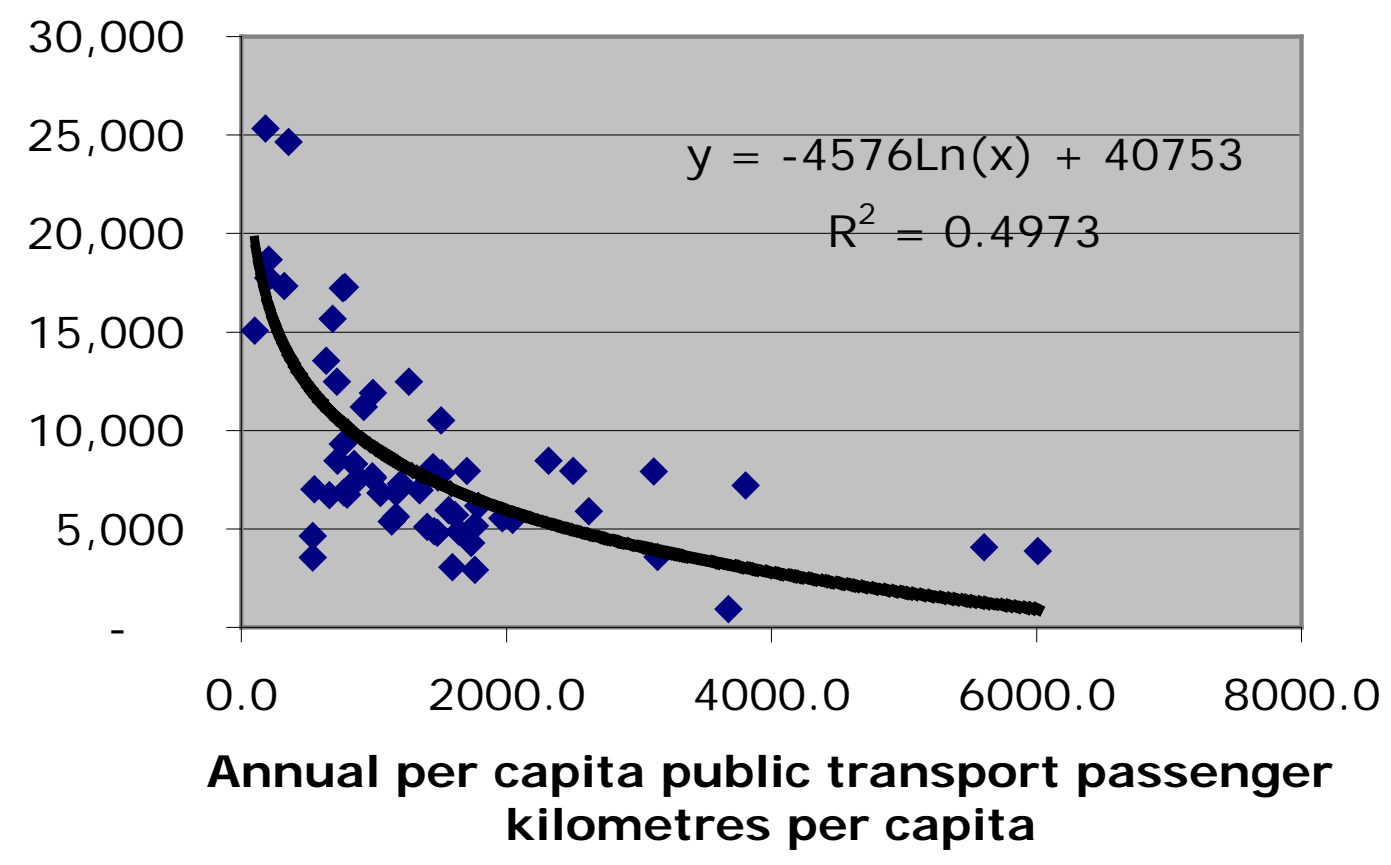

The data on private transport use and public transport use in selected Australian cities for 1996 is given in Table 1 (passenger kilometres per capita in each case).

\begin{tabular}{|l|l|l|}
\hline City & $\begin{array}{l}\text { Private transport use } \\
\text { (pass kms/person) }\end{array}$ & $\begin{array}{l}\text { Public transport use } \\
\text { (pass kms/person) }\end{array}$ \\
\hline Sydney & 10506 & 1509 \\
\hline Melbourne & 11918 & 994 \\
\hline Brisbane & 12487 & 720 \\
\hline Perth & 13546 & 642 \\
\hline
\end{tabular}

Table 1 Car and public transport use per capita in four Australian cities, 1996 
These values in Figure 2 show Australian cities are somewhat down the curve from the very high US cities, which have almost no transit (some around the 100 to 200 pass kms per person) and very high private transport use of over 15,000 pass $\mathrm{kms}$ per person.

The data show that the highest Australian city Sydney had $12.3 \%$ of its total motorised pass kms on transit and that the lowest was Perth with $4.5 \%$ (this was before the remarkable increase in patronage associated with Perth's rail revival).

If Sydney doubled its transit use to 3018 pass kms per person it would from Figure 2 have a per capita private transport use of 4088 passenger kms per capita which is a $61 \%$ reduction in car passenger kms per person over the 1996 figure. If Perth was able to continue the rapid growth in transit patronage and triple its 1996 use to around 2000 pass kms per person then it would reduce its private transport use per capita to 6000 car passenger kms per capita, which is a reduction of 56\% over the 1996 level. Similar calculations can be done for the other Australian cities. Indeed it is feasible that each city could set a target of increases in passenger kms per capita for public transport in order to achieve certain target reductions in car use as part of their commitment to reaching the national goal of $80 \%$ reduction in greenhouse gases by 2050 .

These remarkable reductions suddenly become imaginable. But are they real? Could it happen?

The driving force would need to be a combination of push and pull. The push would come from fuel prices that rise inevitably as supply of oil declines and other alternative fuels just cannot fill the gaping hole in supply. In the US in the past year where fuel price rises have been more severe (as Australia was shielded by the rising dollar), there has been a reduction in VKT of $4.3 \%$ and a substantial rise in transit patronage.

This trend cannot continue unless there is a simultaneous pull from the provision of transit. Already capacity limits have been reached across Australian cities in their public transport so for a start substantial increases in trains, trams and buses are needed to fill the rapid growth in transit. There will also need to be new lines and new technology like Metros and light rail to increase the capacity and speed of transit to make it attractive to use.

At the same time the cities will need to develop rapidly around transit stations. This can be a significant source of funding for the required rail infrastructure through 'Value Transfer PPPs' as in the very successful Chatswood Transport Interchange PPP which has created a new railway station and bus interchange along with a retail and residential complex that makes a small city around and over the station. It can be the main mechanism for replacing the development of car dependent suburbs which are already beginning to die as the price of fuel climbs. Significant new local transit options linking across the heavy rail corridors - especially with light rail systems - will also be needed.

It is important to note here that passenger kms by public transport has two components. The first is boardings and the second is the average distance a boarding is carried. It is clearly more desirable to increase transit usage by increasing use of the system, rather than the same level of patronage just travelling further. The strategy outlined here would tend to achieve this by ensuring that more people 
are clustered around transit stations so that more people use the system and because development is more centred, trip distances and hence travel times would tend to be minimised.

How realistic is it to assume public transport can increase as described, and what are the capacity implications of such an assumption for our public transport systems?

Table 2 below shows the medium population projections for the five largest Australian cities to 2051 (ABS 3222.0). As can be seen, these reveal that:

- The five largest cities are expected to grow by around $20 \%$ between 2004 and 2021 , and by $45 \%$ by 2051

- They will increase their share of Australia's population slightly from $61 \%$ to $63 \%$ over that time

- Although all cities will grow, Brisbane is expected to grow the fastest (almost $90 \%$ growth by 2051) and Adelaide the slowest.

Table 2: Medium Population Projections for Australia, 2004 - 2051

\begin{tabular}{|l|c|c|c|c|c|}
\hline & & & & Growth \% & $\begin{array}{c}\text { Growth \% } \\
\mathbf{2 0 0 4}- \\
\text { City }\end{array}$ \\
\hline Sydney & $\mathbf{2 0 0 4}$ & $\mathbf{2 0 2 1}$ & $\mathbf{2 0 5 1}$ & $\mathbf{2 0 0 4 - 2 0 2 1}$ & $\mathbf{2 0 5 1}$ \\
Melbourne & 4225 & 4871 & 5608 & $15 \%$ & $33 \%$ \\
Brisbane & 3593 & 4252 & 5041 & $18 \%$ & $40 \%$ \\
Perth & 1778 & 2404 & 3355 & $35 \%$ & $89 \%$ \\
Adelaide & 1455 & 1875 & 2454 & $29 \%$ & $69 \%$ \\
Rest of Aust & 1123 & 1201 & 1203 & $7 \%$ & $7 \%$ \\
\hline Australia & $\mathbf{7 9 1 7}$ & 9268 & 10509 & $17 \%$ & $33 \%$ \\
\hline Five City Sub- & $\mathbf{2 0 0 9 1}$ & $\mathbf{2 3 8 7 1}$ & $\mathbf{2 8 1 7 0}$ & $\mathbf{1 9 \%}$ & $\mathbf{4 0 \%}$ \\
Total & $\mathbf{1 2 1 7 4}$ & $\mathbf{1 4 6 0 3}$ & $\mathbf{1 7 6 6 1}$ & $\mathbf{2 0 \%}$ & $\mathbf{4 5 \%}$ \\
\hline \% in 5 largest cities & $61 \%$ & $61 \%$ & $63 \%$ & & \\
\hline
\end{tabular}

Source: ABS 32220.0: Population Projections 2004 - 2101

Table 3 below shows the implications in terms of per capita passenger-kilometres in those cities ranging from a doubling by 2051 for Sydney to a tripling for the small cities (Brisbane, Adelaide and Perth) as suggested in the analysis above. Thus they suggest per capita public transport use in Melbourne in 2021 would be slightly above that achieved in Sydney in 2004, while Perth and Adelaide's use in 2051 would equal that of Sydney currently.

Table 3. Assumed per-capita public transport use in Major Australian Cities (pass kms per year)

\begin{tabular}{|l|c|c|c|c|}
\hline City & $\mathbf{1 9 9 6}$ & $\mathbf{2 0 0 4}$ & $\mathbf{2 0 2 1}$ & $\mathbf{2 0 5 1}$ \\
\hline Sydney & 1509 & 1500 & 2100 & 3000 \\
Melbourne & 994 & 990 & 1600 & 2500 \\
Brisbane & 720 & 800 & 1300 & 2200 \\
Perth & 642 & 700 & 1200 & 2000
\end{tabular}


The total public transport travel task implied by these predictions is shown in table 4 combining the derived per capita growth figures with the predicted population increases. This shows that across the five largest cities total patronage would need to be lifted by $80 \%$ by 2021 , and more than trebled by 2051.

Table 4: Implications for Overall Public Transport Use

Table 3: Estimated Pass-kms (Billion)

\begin{tabular}{|l|c|c|c|c|c|}
\hline City & $\mathbf{2 0 0 4}$ & $\mathbf{2 0 2 1}$ & $\mathbf{2 0 5 1}$ & $\mathbf{2 0 0 4 - 2 0 2 1}$ & $\begin{array}{c}\text { Growth \% } \\
\mathbf{2 0 0 4} \mathbf{2 0 5 1}\end{array}$ \\
\hline Sydney & 6.3 & 10.2 & 16.8 & $61 \%$ & $165 \%$ \\
Melbourne & 3.6 & 6.8 & 12.6 & $91 \%$ & $254 \%$ \\
Brisbane & 1.4 & 3.1 & 7.4 & $120 \%$ & $419 \%$ \\
Perth & 1.0 & 2.3 & 4.9 & $121 \%$ & $382 \%$ \\
Adelaide & 0.6 & 1.0 & 1.8 & $71 \%$ & $221 \%$ \\
\hline Total & $\mathbf{1 2 . 9}$ & $\mathbf{2 3 . 4}$ & $\mathbf{4 3 . 5}$ & $\mathbf{8 1 \%}$ & $\mathbf{2 3 7 \%}$ \\
\hline
\end{tabular}

However the increase in patronage in peak periods would not need to be as large as in off-peak periods, given the much lower share achieved for non-work or education trips (such as social/recreation, shopping and business trips) which are largely made in off-peak periods. This is shown in table 5 below to illustrate the task in terms of augmenting public transport capacity at peak periods in each of the cities to achieve the above increase in public transport use.

Table 5. Estimated Increase in Peak and Off-Peak Capacity

\begin{tabular}{|l|c|c|c|c|}
\hline \multirow{2}{*}{ City } & \multicolumn{2}{|c|}{ Growth \% } & 2004-2021 & \multicolumn{2}{c|}{ Growth \% } & 2004-2051 \\
\cline { 2 - 5 } & Peak & Off-Peak & Peak & Off-Peak \\
\hline Sydney & $\mathbf{5 0 \%}$ & $70 \%$ & $\mathbf{1 2 0 \%}$ & $200 \%$ \\
Melbourne & $\mathbf{7 0 \%}$ & $110 \%$ & $\mathbf{2 0 0 \%}$ & $300 \%$ \\
Brisbane & $\mathbf{1 0 0 \%}$ & $140 \%$ & $\mathbf{3 0 0 \%}$ & $500 \%$ \\
Perth & $\mathbf{1 0 0 \%}$ & $140 \%$ & $\mathbf{2 8 0 \%}$ & $480 \%$ \\
Adelaide & $\mathbf{5 0 \%}$ & $90 \%$ & $\mathbf{1 5 0 \%}$ & $300 \%$ \\
\hline Total & $\mathbf{6 5 \%}$ & $\mathbf{9 5 \%}$ & $\mathbf{1 6 0 \%}$ & $\mathbf{3 2 0} \%$ \\
\hline
\end{tabular}

Hence to achieve major reductions in car use it would be necessary to increase capacity in Sydney by around $50 \%$ by 2021 , and by $120 \%$ by 2051 . For Brisbane the increases are more like a doubling in capacity by 2021 and a quadrupling by 2051. These are not difficult to imagine as they represent growth rates of around $2 \%$ per year.

With such growth the transformation of Australian cities to achieve significant reductions in car use can then happen. Data on how this can happen based on each Australian city is provided in the companion paper by Gary Glazebrook: 'Scope for Enhancing Public Transport'. 


\section{Conclusions}

The growth of public transport in Australian cities to 2050 is mapped out to show that doubling in the major cities and trebling in the smaller cities is a feasible target. The increase from around 1500 pass $\mathrm{kms}$ per person to 3000 pass kms per person in Sydney would be associated with a reduction in per capita vehicle use from 10,400 pass kms to 4,800 pass $\mathrm{kms}$, i.e. a reduction of $61 \%$, due to the transit leverage effect as indicated by the data from over 100 cities in a global survey. The same process in Perth would mean an increase in public transport from 640 pass kms to 2000 pass kms which would be associated with a decline per capita car use from 13,500 pass kms to 6000 pass kms i.e. a reduction of $56 \%$. These kinds of changes are only imaginable if the price of fuel continues to stay high (more than likely) and the public transport agencies are able to compete for infrastructure funding (hopefully possible). At the very least it gives a hopeful perspective that Australian cities could use the present crisis and opportunity provided by the fuel situation to make our cities much more sustainable.

\section{References}

Newman P and Kenworthy J (1999) Sustainability and Cities: Overcoming Automobile Dependence, Island Press, Washington DC.

Newman P, Beatley T and Boyer H (2008) Resilient Cities: Responding to Peak Oil and Climate Change, Island Pres, Washington DC. 